\title{
Genesis
}

Manuscrits - Recherche - Invention

41 | 2015

Créer à plusieurs mains

\section{Do you know... ? et Thinking together. Observer les formes de l'action collective}

- H. S. Becker, R. R. Faulkner, «Qu'est-ce qu'on joue, maintenant ? » Le répertoire de jazz en action, trad. Bruno Gendre, Paris, La Découverte, 2011 [2009], 298 p.

- H. S. Becker, R. R. Faulkner, Thinking Together. An E-mail Exchange and All That Jazz, Paris, Les laboratoires d'Aubervilliers et Questions théoriques, 2013, $350 \mathrm{p}$.

\section{Irina Kirchberg}

\section{OpenEdition}

\section{Journals}

Édition électronique

URL : http://journals.openedition.org/genesis/1558

DOI : 10.4000/genesis. 1558

ISSN : 2268-1590

Éditeur :

Presses universitaires de Paris Sorbonne (PUPS), Société internationale de génétique artistique littéraire et scientifique (SIGALES)

\section{Édition imprimée}

Date de publication : 27 novembre 2015

Pagination : 160-162

ISBN : 9791023105049

ISSN : 1167-5101

\section{Référence électronique}

Irina Kirchberg, «Do you know... ? et Thinking together. Observer les formes de l'action collective » Genesis [En ligne], 41 | 2015, mis en ligne le 03 mai 2017, consulté le 22 septembre 2020. URL : http:// journals.openedition.org/genesis/1558; DOI : https://doi.org/10.4000/genesis.1558 


\section{Observer les formes de l'action collective}

\section{- Howard S. Becker, Robert R. Faulkner, «Qu'est-ce qu'on joue, maintenant? » Le répertoire de jazz en action, trad. Bruno Gendre, Paris, La Découverte, 2011 [2009], 298 p.}

\section{- Howard S. Becker, Robert R. Faulkner, Thinking Together. An E-mail Exchange and All That Jazz, éd. Dianne Hagaman, Paris, Les laboratoires d'Aubervilliers/Questions théoriques, 2013, 350 p.}

\section{Compte rendu par Irina Kirchberg}

Tout au long de leur carrière, Howard S. Becker et Robert R. Faulkner ont trouvé dans le travail artistique un terrain d'étude particulièrement pertinent pour, tout à la fois, vérifier et affiner leur conception de l'organisation des mondes sociaux ${ }^{5}$. Ces deux auteurs, sociologues et musiciens, s'inscrivent dans la tradition de la seconde école de Chicago qui porte son attention sur « l'assemblage progressif des actions individuelles qui produit une action collective cohérente » $\left(D y K^{6}\right.$, p. 266). Pour eux, chacune des actions posées par les individus résultent de la construction de sens qu'ils opèrent à partir de leurs interactions avec d'autres acteurs ("people are forever creating the cultural understanding on which collective action depends », TT7, p. 133) et ne peut se réduire mécaniquement à des structures préexistantes (ce qui serait plus proche d'une conception dispositionnaliste de l'action ${ }^{8}$ ).

Dans Qu' est-ce qu'on joue maintenant, Howard S. Becker et Robert R. Faulkner, respectivement pianiste et trompettiste, mettent à profit leur connaissance intime des mondes du jazz ${ }^{9}$ (de son langage musical, de ses lieux et de ses codes et ses « gens ») pour bâtir leur réflexion sur la façon dont se constitue le répertoire de standards de jazz d'une soirée.

Résidant de part et d'autre des États-Unis, les auteurs ont discuté de cette thématique et en ont posé les premiers jalons via des échanges de courriels quasiment journaliers entre 2003 et 2005. Publié en 2013, l'ouvrage Thinking Together, foisonnant d'anecdotes personnelles, de références sociologiques et de renvois à des interprétations musicales ${ }^{10}$, rassemble cette correspondance qui portait en germe la trame de Do you know... Drôle d'effet de mise en abyme que nous permet d'observer l'édition successive et chronologiquement inversée de ces deux ouvrages. L'un nous permet de suivre très concrètement les processus de choix permanents auxquels se livrent Becker et Faulkner pour donner corps à un livre qui, lui-même, cherche à rendre compte des « ajustements et réajustements » (DyK, p. 267) récurrents auxquels se livrent les musiciens de jazz pour pouvoir assurer un plan (les gigs).

Bien que le projet commun puisse sembler simple (« our concern is with actual situations where some actual people have to do some actual real thing together in a real time and in a real place », TT, p. 132), les mondes du jazz le mettent à

5. On citera notamment les publications suivantes: H. S. Becker, Outsiders. Études de sociologie de la déviance, Paris, Métailié, 1985 [1963] ; H. S. Becker, Les Mondes de l'art, Paris, Flammarion, 1988 [1982] ; R. Faulkner, Music on Demand: Composers and Carreers in the Hollywood Film Industry, New Brunswic, Transaction Book, 1983 ; R. Faulkner, Hollywood Studio Musicians: Their Work and Carreers in the Film Industry, Chicago, Aldin-Athertone, 1971.

6. Cette abréviation fait référence au titre de la publication originale de l'ouvrage "Qu'est-ce qu' on joue maintenant? " Le répertoire jazz en action, intitulé dans sa version initiale : Do You Know... ? The jazz Repertoire in Action. H. S. Becker, R. R. Faulkner, "Qu'est-ce qu' on joue, maintenant? » Le répertoire de jazz en action, Paris, La Découverte, 2009 (trad. B. Gendre) ; Do You Know... ? The jazz Repertoire in Action, Chicaco, Chicago University Press, 2011.

7. Nous renvoyons ici à l'ouvrage d'Howard S. Becker et Robert R. Faulkner : Thinking Together. An E-mail Exchange and All That Jazz, Paris, Les laboratoires d'Aubervilliers et Questions théoriques, 2013.

8. Pour les chercheurs inscrits dans ce courant, l'action résulte des rapports de force qui s'établissent entre des dispositions individuelles (les inclinaisons à penser, sentir ou agir incorporées par les individus durant leur socialisation) et les éléments de contexte (qui activent, inhibent ou mettent en veille certaines d'entre elles). À ce propos, on pourra consulter l'ouvrage de B. Lahire, L'Homme pluriel. Les ressorts de l' action, Paris, Hachette, 2006 [1998].

9. Robert Faulkner est, encore à l'heure actuelle, trompettiste de jazz, et Howard Becker a payé ses études en étant pianiste dans les bars. Tous deux font état de l'importance de leur connaissance «pratique » de ce terrain (ce qui est courant de la part des sociologues interactionnistes adeptes de l'observation participante) mais, plus important ici, explicitent dans un appendice de l'ouvrage (de même que dans un article de 2008) l'intérêt de la méthode ethnographique dans le cadre de l'observation des pratiques artistiques et plus spécifiquement musicales. Lire R. Faulkner et H. S. Becker, « Studying Something You Are Part Of: The View From The Bandstand », Ethnologie française, vol. 38, 2008, p. 15-21.

10. La version numérique de Thinking Together donne également accès aux liens YouTube des très nombreuses références musicales mobilisées par les deux correspondants lors de leurs échanges. Ce procédé offre l'avantage de restituer la matière sonore même de l'objet de cette étude. 
rude épreuve. Les conditions dans lesquelles sont élaborées les set lists (listes des morceaux interprétés) des soirées sont en effet singulières au regard des modes d'activités généralement développés par les artistes d'autres disciplines : ces jazzmen ne se connaissent pas toujours, jouent sans répétition ni partition, et se produisent dans des lieux différents chaque soir (bar, salle de concert, engagement privé). Becker et Faulkner s'étonnent donc : que peuvent bien avoir en commun ces musiciens qui leur permet de jouer ensemble de façon satisfaisante ? Ces deux chercheurs ne sont pas les seuls à s'interroger à ce sujet et les jazzmen eux-mêmes débutent bien souvent leurs soirées en se posant cette question : « Tu connais [tel morceau]... ?» (Do you know...? ?)11. Chacun s'attelle à jauger l'ensemble des « standards » parmi lesquels il sera possible de puiser pour élaborer, sur le champ, le programme de la prestation. Il s'agit de trouver un point de concordance entre le « répertoire individuel» de chacun des musiciens (dépendant de l'âge, du parcours, de la curiosité) et ce qui peut être joué en fonction de la situation («We're playing a bar mitzvah, so "What A Friend We Have in Jesus", while a fun tune to play, does not fit us on this occasion », TT, p. 98)12. S'ensuit une succession d'étapes faites de questions-réponses des musiciens entre chaque morceau et d'ajustement aux réactions des patrons et du public. L'élaboration du répertoire musical d'une soirée se constitue au fil de micronégociations dont Howard $\mathrm{S}$. Becker et Robert R. Faulkner s'attachent à faire apparaître les enjeux. En amont de la prestation, il s'agit donc de ratifier ou d'évincer un standard du programme de la soirée tout en affirmant sa compétence de musicien. Difficile, en effet, pour un des interprètes d'avouer qu'il ne connaît pas un titre sans écorner ce qui fait partie de la compétence professionnelle d'un musicien ordinaire $(D y K, \text { p. } 30-31)^{13}$ et risquer ainsi de perdre la face vis-à-vis de ses collègues. Le mieux est encore de proposer, du tac au tac, une autre référence ou, de façon plus risquée, de se lancer, coûte que coûte, dans l'interprétation de ce morceau en se fiant à ses réflexes de musicien.

Ces moments de flottement font apparaître tous les points d'accroche qui servent de support à la coordination d'une action collective. C'est la connaissance du langage musical du jazz, de la régularité de ses grilles, de la logique des enchaînements harmoniques, mais également des petits gestes du métier ( $D y K$, p. 221) qui permettent aux musiciens de se lancer quotidiennement dans l'aventure de l'interprétation et d'infléchir, sur le vif, la forme de leurs improvisations. Ainsi apparaissent les dimensions conjointement esthétiques et pratiques des conventions qui président au bon déroulement de l'action collective.

Si ces analyses donnent à voir tout le fonds commun de savoir et de savoir-faire nécessaire pour coordonner des activités de création, les auteurs montrent également que l'action collective ne se résume pas à puiser dans ce qui ne serait alors qu'une boîte à outils de savoir-faire : « one thing that's wrong with the idea of "tool kit" is that it doesn't really leave room for adding to, deleting, forgetting, and all the other operations that change its content, which as we know now are all so juicy, and all add a layer of complexity und uncertainty to anything involving a "tool kit" » (TT, p. 130). Dans Do You Know... ? (comme dans Thinking Together), on voit le processus par lequel chacun des musiciens (ou sociologues !) essaie de satisfaire ses propres envies, d'affirmer ses opinions tout en se mettant au service du projet commun.

Ainsi se résout l'intrigue amorcée dans le chapitre introductif de Do You Know... ? par la présentation, extrêmement contrastée, de trois soirées ayant eu lieu à trente ans d'intervalles. Les six chapitres centraux de cet ouvrage révèlent les multiples natures du « répertoire », entre corpus individuel de standards en perpétuelle évolution au fil de la

11. Puis leurs collègues demandent des précisions sur la tonalité, la grille ou la rythmique (en ré ? un blues ? style bossa nova ?) et étudient la proposition (connaissent-ils ce titre ? sont-ils capable de le jouer ? ont-ils envie de le jouer?).

12. L'analyse dépasse ainsi les bornes des seuls artistes pour s'ouvrir à d'autres intervenants et montrer comment ceux-ci prennent part au processus de création en cours sur scène. Les personnes qui ont engagé le groupe (patrons de bar, agent artistique) et les spectateurs pour qui les jazzmen jouent en dernière instance ont des désirs et des intérêts que ces musiciens doivent souvent satisfaire sous peine de ne plus être engagés. 13. Howard S. Becker et Robert R. Faulkner optent pour le vocabulaire employé par Marc Perrenoud. Dans son ouvrage, Les Musicos, ce dernier décrit les conditions de travail des musiciens ordinaires qui « cachetonnent » (c'est-à-dire assurent le plus de dates possibles et ce, dans des styles extrêmement variés), afin de subvenir à leurs besoins. M. Perrenoud, Les Musicos Enquête sur des musiciens ordinaires, Paris, La Découverte, 2007.

14. Robert Faulkner décrit d'ailleurs ce phénomène à partir de son expérience d'instrumentiste : «My repertoire has it has been constituted over the years, and as I am continuing to constitute it, is continuing to grow, that it's a living thing, not a thing that just sits here like an already constituted lump » (TT, p. 311). 
carrière du musicien (le répertoire individuel analysé dans les chapitres III et IV) ${ }^{14}$, fonds commun spécifique à une époque dans lequel il s'agit de puiser (chapitres V et VI), et résultat toujours renouvelé de choix opérés collectivement dans des situations spécifiques (le répertoire en action étudié dans les chapitres VII et VIII). On aboutit alors à une conception du répertoire comme activité (chapitre IX) dans laquelle ce que font les interactants n'est « ni entièrement aléatoire et discontinu, ni entièrement fixe et déterminé » (DyK, p. 258).

Cette conclusion trouve une illustration parfaite dans Thinking Together, qui reproduit, dans leur ordre chronologique, les centaines de courriels échangés par Becker et Faulkner pour rédiger Do You Know... ?. Le style même des échanges, entre dynamisme, humour et familiarité rend justice à la posture scientifique de ces deux auteurs et fait la part belle aux «mécanismes ordinaires » (DyK, p. 276) de cette aventure collective. Les solides réflexions conceptuelles de ces deux sociologues sont entrecoupées de passages jalonnés d'hésitations (marquées par les «hum » et les «duh» de Faulkner), de convictions tranchées affirmées avec vigueur (TT, p. 60) et d'interjections hautes en couleur. Le choix éditorial de faire apparaître en en-tête de chaque message le sujet, la date, le nom de l'auteur et du destinataire du message permet, quant à lui, de suivre l'enchevêtrement et la trajectoire des idées proposées par chacun des deux sociologues. Passant de la profusion de messages un jour (six courriels le 15 novembre 2003) aux silences prolongés, de réflexions développées à de simples relances de quelques lignes, de modélisations en tableau à des anecdotes enrichies de références musicales, on saisit les temporalités de ce processus de création, sa matière sonore et le «montage 15 » intellectuel auquel se livrent les deux auteurs pour faire avancer leur réflexion.

Qu'il s'agisse de « jouer » ou de « penser ensemble16», Howard Becker et Robert Faulkner montrent comment, lorsqu'il s'agit de créer à plusieurs mains, l'accord final « se gagne pas à pas » (DyK, p. 204).

15. C'est par ce vocabulaire (« editing ») qu'Howard Becker analyse les successions de choix permanents qui président à la constitution d'une œuvre, H. S. Becker, Les Mondes de l'art, op. cit., p. 192-225.

16. Le nom de cette correspondance éditée (Thinking Together...) fait d'ailleurs référence à l'ouvrage d'Eliot Freidson, Doctoring together. A study of professional Social Control, Chicago, Chicago University Press, 1981. 